\title{
Salud Bucal y Enfermedades no transmisibles en pacientes de un centro de enseñanza universitaria, Montevideo-Uruguay. Parte 1
}

\author{
Oral Health and Non communicable Diseases in patients of a \\ higher education institution, Montevideo, Uruguay 2016. \\ Saúde bucal e doenças não transmissíveis em pacientes de uma \\ instituição de ensino superior, Montevidéu, Uruguai 2016.
}

Susana Margarita Lorenzo-Erro ${ }^{1}$ ORCID: 0000-0003-4801-0761
Estela Skapino² ORCID: 0000-0003-3575-3371
Mariana Musto ${ }^{3}$ ORCID: 0000-0002-4741-7773
Patricia Olmos ${ }^{3}$ ORCID: 0000-0002-6003-1197
Ramón Álvarez ${ }^{1}$ ORCID: 0000-0002-2505-4238
Anunzziatta Fabruccini ${ }^{1}$ ORCID: 0000-0001-7344-4751
Fernando Massa ${ }^{4}$ ORCID: 0000-0002-2922-4097

DOI: 10.22592/ode2020n36a7
Paula Moliterno² ORCID: 0000-0002-7377-9786

Beatriz Vila ${ }^{5}$ ORCID: 0000-0002-6854-5811

Sylvia Piovesán ${ }^{6}$ ORCID: 0000-0003-1807-6380

Laura Cosetti ${ }^{7}$ ORCID: 0000-0001-7282-3362

Florencia Pisón ${ }^{8}$ ORCID: 0000-0002-5614-2329

Romina Pesce ${ }^{9}$ ORCID:0000-0003-2624-257X

Rossina Sanguinetti ${ }^{9}$ ORCID: 0000-0002-7901-512X

María Gardiol ${ }^{9}$ ORCID:0000-0001-9502-8003

\section{Resumen}

Las enfermedades no transmisibles (ENT) comparten factores de riesgo conductuales y metabólicos con las enfermedades bucales y ambas representan un problema de salud pública.

Objetivo: Determinar la prevalencia ENT y sus factores de riesgo conductuales y metabólicos en personas que concurrieron a la Facultad de Odontología de la Universidad de la República.

Métodos: Estudio transversal en el que se aplicó un cuestionario sobre características sociodemográficas y hábitos vinculados a factores de riesgo comportamentales. Se realizaron mediciones antropométricas, de presión arterial y glicemia capilar.

Resultados: Fueron encuestados 602 individuos

Conclusiones: En función de las prevalencias elevadas de varios factores de riesgo, se recomienda la instalación de un programa preventivo-educativo en las salas de espera de la Facultad.

Palabras clave: Enfermedades no transmisibles, Salud Bucal, Epidemiología, Interdisciplinario.

Servicio de Epidemiología y Estadística, Cátedra de odontología Social, Facultad de Odontología, Universidad de la República, Montevideo, Uruguay.

Departamento de Nutrición Clínica, Escuela de Nutrición, Universidad de la República, Montevideo, Uruguay.

Cátedra de Odontología Social, Facultad de Odontología, Universidad de la República, Montevideo, Uruguay.

Instituto de Estadística. Facultad de Ciencias Económicas, Universidad de la República. Montevideo, Uruguay.

Cátedra de Endodoncia, Facultad de Odontología, Universidad de la República. Montevideo, Uruguay.

Servicio de Registro y Admisión de Pacientes, Facultad de Odontología, Universidad de la República, Montevideo, Uruguay.

Cátedra Patología y Semiología Buco Maxilar y Clínica Estomatológica, Facultad de Odontología, Universidad de la República. Montevideo, Uruguay.

8 Facultad de Enfermería, Universidad de la República, Montevideo, Uruguay.

9 Práctica privada. Facultad de Odontología, Universidad de la República, Montevideo, Uruguay.

Fecha de recibido: 12/11/2019 - Fecha de aceptado: 10/3/2020 


\section{Abstract}

Non-communicable diseases (NCD) share behavioral and metabolic risk factors with oral diseases and both represent a public health problem.

Objective: to find out the prevalence of NCD and its behavioral and metabolic risk factors in people who attend at College of Odontology of the University of the Republic.

Methods: A Cross-sectional study was conducted and a questionnaire related to socio-demograhic characteristics and habits linked to behavioral risk factors was applied. Anthropometric measurements of arterial pressure and capillary glucose were taken.

Results: The final sample were 602 individuals. A high prevalence of physical inactivity, problematic alcohol intake, excessive salt consumption, hypertension and diabetes was found. These figures were larger than those found at the national level.

Conclusions: Due to the high prevalence of various risk factors, the installation of a preventive-educational program in the waiting rooms of the Faculty is recommended.

Keywords: Non-communicable diseases, Oral Health, Epidemiology, Interdisciplinary.

\section{Introducción}

Las enfermedades no transmisibles (ENT) son un conjunto de patologías vinculadas al estilo de vida moderno que pueden explicarse al analizar el fenómeno de transición demo-epidemiológica. Este fenómeno se constata cuando en la década del veinte se pasa de un modelo de influencia cultural agrícola, donde las causas de muerte principales son las enfermedades infecciosas, a otro modelo de sociedad industrial y posindustrial, donde las causas principales de muerte son las $\mathrm{ENT}^{(1,2)}$. Es así que este nuevo

\section{Resumo}

As doenças não transmissíveis (DNT) compartem fatores de risco comportamentais e metabólicos com as doenças bucais, as duas representam um problema de saúde pública. Objetivo: Determinar a prevalência DNT e os fatores de risco comportamentais e metabólicos em pessoas que concorreram à $\mathrm{Fa}$ culdade de Odontologia da Universidade da República.

Métodos: Foi realizado um estudo transversal, onde foi aplicado um questionário relativo às características sociodemográficas e hábitos ligados com fatores de risco comportamentais. Se realizaram mediçóes antropométricas, de pressão arterial e glicemia capilar.

Resultados: Foram pesquisados 602 indivíduos. Constatou-se uma alta prevalência de inatividade física, ingestão problemática de álcool, consumo excessivo de sal, hipertensão e diabetes. Estes valores foram acima dos encontrados a nível nacional.

Conclusáo: Dada a alta prevalência encontrada de vários fatores de risco, recomendase a instalação de um programa educacional -preventivo nas salas de espera da Faculdade.

Palavras-chave: Doenças não comunicáveis, Saúde Oral, Interdisciplinar.

modelo productivo, conforma el nivel de determinación general de la salud y la enfermedad, influenciando el nivel particular de los modos de vida de los grupos sociales, que a su vez determina el nivel individual donde se expresan los estilos de vida de las personas ${ }^{(3)}$.

Las ENT que generan la mayor carga de mortalidad a nivel mundial son fundamentalmente cuatro: enfermedades cardiovasculares (ECV), cáncer, enfermedades respiratorias crónicas y diabetes. El 75\% de las muertes por estas patologías se produce en países con ingresos medios 
y bajos $^{(4)}$. En Uruguay las ENT determinan casi 7 de cada 10 muertes registradas ${ }^{(5)}$. Si bien estas patologías se asocian con edades avanzadas, existe un número creciente de personas menores de 70 años que fallecen por estas enfermedades, sobre todo en las regiones más pobres. ${ }^{(6)} \mathrm{El}$ enfoque epidemiológico clásico establece que estas enfermedades comparten cuatro factores de riesgo comportamentales: alimentación inadecuada (dieta alta en sodio, azúcar refinado, y grasas saturadas y trans y además baja en fibras), tabaquismo, consumo nocivo de alcohol e inactividad física. La exposición a estos factores de riesgo conductuales, determina que con el tiempo se desarrollen factores de riesgo metabólicos: obesidad, hipertensión arterial, glicemias de ayuno elevadas, colesterol elevado. Los factores de riesgo comportamentales y metabólicos son pasibles de ser modificados, haciendo que estas patologías sean prevenibles, representando una disminución importante en el número de muertes prematuras ${ }^{(7)}$. Las patologías bucales como la enfermedad periodontal, caries, inflamación de la mucosa y pérdida dental si bien no presentan alta mortalidad, son un componente de la carga global de enfermedades crónicas y comparten muchos de los factores de riesgo de las $\mathrm{ENT}^{(8)}$, tanto es así que desde el año 2011 las enfermedades bucales, junto con las renales y oculares, han sido incluidas en el Informe sobre ENT en su artículo $19^{(9)}$.

Según Watt R. (2012) ${ }^{(10)}$ el enfoque de factores comunes de riesgo, reconoce que existen factores de riesgo compartidos entre las ENT y las enfermedades bucales, lo cual permitiría un trabajo conjunto más eficiente para la salud pública. Por otro lado un enfoque epidemiológico más amplio, como es la determinación social de la salud, establece ciertas discrepancias con el enfoque de riesgo, cuestionando su capacidad explicativa integral. Autores como Breilh ${ }^{(3)}$, enmarcados en el paradigma de la determinación social, plantean que referirse a "factores de riesgo" implica un enfoque fragmentado de la realidad ya que los "factores" sólo capturan una par- te de la compleja causalidad de los procesos de salud y enfermedad, descontextualizándolos y desvinculándolos de los niveles de mayor jerarquía causal (modos de vida y estructura social), lo cual no permite comprender el mecanismo de producción y desarrollo de estas patologías. Existen escasos estudios relativos al abordaje conjunto de los factores de riesgo para enfermedades no transmisibles en el Uruguay (11) de acuerdo a la Segunda Encuesta Nacional de Factores de Riesgo de ENT ${ }^{(12)}$ llevada a cabo por el Ministerio de Salud Pública de Uruguay, el 50\% de la población adulta del país tiene 3 o más factores de riesgo combinados. NO se han encontrado estudios que adopten el enfoque común de factores de riesgo para enfermedades bucales y ENT a nivel nacional. El presente trabajo tuvo como objetivo conocer las características socio-demográficas, la prevalencia de factores de riesgo de las ENT y las enfermedades bucales más frecuentes (caries y paradenciopatías) en usuarios que demandan atención en la Facultad de Odontología de la UDELAR. En esta primera parte se presentarán sólo los resultados de la prevalencia de ENT en los pacientes de la FO.

\section{Metodología}

Se trató de un estudio descriptivo, transversal, realizado en el Servicio de Registro y Admisión de la Facultad de Odontología en el período agosto 2015 - mayo 2016.

La estrategia de muestreo utilizada tuvo en cuenta la estacionalidad de la concurrencia de las personas a registrarse en la Facultad de Odontología y los tres turnos en que funciona el Servicio de Registro (mañana, tarde y noche). El trabajo de campo se realizó entre los meses de agosto a noviembre (2015) y marzo a mayo (2016). Por tal motivo durante el período de recolección de datos el reclutamiento de los pacientes siguió una lógica de muestreo secuencial, donde participaban todos los pacientes que estaban citados en ese turno y en ese día. Por lo 
tanto, dada esa logística que el equipo de investigación consideró la más apropiada en términos de recursos humanos disponibles, el diseño finalmente empleado es un diseño mixto, dado que es secuencial pero donde de antemano se tenía fijado un tamaño de muestra previamente calculado de 800 al inicio del estudio. Estel tamaño de muestra surge de considerar el hacer una muestra bajo un diseño asimilable al Muestreo aleatorio Simple, el que permite hacer estimaciones para prevalencias de hasta un $30 \%$ con una confianza del $95 \%$ y un error del 5\%, considerando hasta dos dominios de estimación (por sexo, o tramo etario), siendo de tipo autoponderado. Estos requerimientos de márgenes de error, confianza y niveles de prevalencias implicó alrededor de 320 por dominio con lo cual fueron necesarios 640 pacientes. Suponiendo una tasa de no respuesta del $20 \%$ se obtuvo el número final de los 800 originalmente planteados.

\section{1) Recolección de datos:}

Los participantes respondieron un cuestionario sobre aspectos sociodemográficos (sexo, edad, estudios cursados, ingresos mensuales, tipo de trabajo y cobertura asistencial en salud), factores de riesgo comportamentales y metabólicos. Además se registraron medidas antropométricas(peso, talla y circunferencia de cintura), glicemia capilar, presión arterial e información referida a las patologías bucales (caries, paradenciopatías, lesiones de mucosa y pérdida dentaria). La talla fue medida con un tallímetro de pie (Marca SECAR), con el individuo descalzo, de pie, de espaldas a la barra medidora, talones juntos, en contacto con el tallímetro. La medida se tomó después de una inspiración profunda manteniendo la cabeza en el plano de Frankfort. La circunferencia de cintura se midió bordeando la circunferencia abdominal al nivel del plano que se encuentra en el punto medio entre el reborde costal y la cresta ilíaca, utilizando una cinta métrica inextensible. La glicemia se midió a través de una punción capilar utilizando lancetas descartables y tiras reactivas para su posterior medición en un glucómetro digital (marca Bayer Contour TS $\mathrm{tm}$ ) siguiendo las recomendaciones del fabricante. Para la medición de la Presión Arterial (PA), se utilizó un aparato aneroide (marca RIESTERR verificado por LATU No 333075. 06/2015). Requirió de una preparación de los participantes a quienes se les solicitó previo a la medición, sentarse tranquilamente durante 15 minutos y se seleccionó el brazalete a utilizar de acuerdo al perímetro de su brazo. Las patologías bucales fueron relevadas a través de un examen odontológico detallado. El mismo se realizó en sillón odontológico, con espejo, pinza y sonda CPI, se registró caries de corona y raíz, luego bolsa periodontal en todos los dientes presentes (manual OMS, 2013) ${ }^{(13)}$, pérdida de inserción por sextante.

\section{2) Aspectos éticos:}

De acuerdo a la normativa vigente en el país el proyecto de investigación fue presentado y aprobado por el Comité de Ética de Investigación de la Facultad de Odontología. Durante la ejecución del relevamiento a partir del diálogo y la comprensión de la investigación que se llevaba a cabo, los individuos a encuestar decidían o no participar de la investigación. De acceder a ello se solicitaba su firma en un formulario de Consentimiento Informado escrito. Los participantes, fueron informados de los resultados de las mediciones obtenidas (peso, talla, circunferencia de cintura, valor glicemia capilar y presión arterial) y asesorados sobre la necesidad de consulta médica cuando correspondía.

\section{3) Entrenamiento y Calibrado:}

Se realizó una estandarización de criterios para la realización del cuestionario (4 reuniones conjuntas entre los diferentes profesionales participantes del estudio), finalmente se elaboró un instructivo de registro de las preguntas correspondientes a los formularios de investigación y se capacitó a los encuestadores para la utiliza- 
ción de las diferentes cartillas (alimentos, bebidas alcohólicas y actividad física).

Se realizó una capacitación en antropometría, donde se explicó la forma de armado del tallímetro de pie y su colocación. El registro de talla se realizó midiendo al mismo individuo en dos oportunidades y en caso de encontrar una diferencia mayor a $0.5 \mathrm{~cm}$ se realizó una tercera medición, promediándose las dos más cercanas. Lo mismo ocurrió para la medida de la circunferencia de cintura. La medida del peso, al realizarse en balanza electrónica no requirió de estandarización (por su característica de lectura directa del propio visor).

Se entrenó a los encuestadores en la punción capilar para la toma de la glicemia y el manejo del glucómetro. Se utilizó un equipo digital con sus correspondientes tiras reactivas y lancetas para punción descartable. Para la medición de la glicemia capilar se siguió el procedimiento correspondiente y las recomendaciones del fabricante.

En cuanto al calibrado para la medición de la PA, se midió la presión en diez individuos en tres ocasiones por tres diferentes operadores. Obteniéndose un grado de concordancia y el margen de error que tenía cada encuestador en la medición de la PA aceptable, con un coeficiente de correlación intraclase entre 0,73-0,91. Para el entrenamiento y calibrado de las patologías bucales, el equipo de trabajo tomó como referencia el Manual de Encuestas Básicas de la OMS ${ }^{(13)}$ del año 2013 y el Relevamiento $\mathrm{Na}$ cional de Salud Bucal realizado en Uruguay en el año $2011^{(14,15)}$.

\section{4) Variables e indicadores:}

Variables comportamentales: Consumo de frutas y verduras: se consideró insuficiente un consumo menor de 5 porciones al día. Consumo de alcohol: se elaboró una variable considerando la frecuencia y número de tragos, con las siguientes categorías: "no consume", "consumo muy bajo", "consumo bajo", "intermedio", "alto" y "muy alto". Se consideró consumo excesivo a las categorías "alto" y "muy alto". Consumo de tabaco: Se consideró factor de riesgo el consumo diario de tabaco. Actividad física: Se consideró actividad física insuficiente: la realización de menos de 75 minutos por semana de actividad física vigorosa o menos de 150 minutos por semana de actividad física moderada. Consumo elevado de sodio: Se consideró factor de riesgo la frecuencia "siempre" y "casi siempre" en que se agrega sal a la comida previamente salada en su elaboración.

Para los factores de riesgo metabólicos se consideró: Hipertensión arterial una medición de Presión arterial sistólica (PAS) mayor o igual a $140 \mathrm{mmHg}$ y/o Presión arterial diastólica (PAD) mayor o igual a $90 \mathrm{mmHg}{ }^{(11)}$, o el autoreporte de ser hipertenso. Se consideró Diabetes / Glicemia alterada en ayunas (GAA)a quienes tenían glicemia capilar mayor o igual a $200 \mathrm{mg} /$ dl con o sin ayuno; o antecedentes de diabetes diagnosticada por su médico y proporcionado a través del autoreporte del paciente (MSP,2007) (11).

Estado nutricional alterado: Circunferencia de cintura alterada en mujeres mayor a $88 \mathrm{~cm}$ y varones mayor a $102 \mathrm{~cm}$. Índice de masa corporal (relación entre el peso $(\mathrm{kg})$ sobre el cuadrado de la talla(m2) - IMC): sobrepeso: 25 a 29, 9kg/ $\mathrm{m} 2$; obesidad: $30 \mathrm{~kg} / \mathrm{m} 2$ o más ${ }^{(16)}$.

Para las patologías bucales, en cuanto a Caries se utilizó como indicador CPOD y para Paradenciopatías se utilizó proporción de bolsa periodontal y pérdida de inserción (PIP).

\section{Resultados}

Se examinaron 602 pacientes. Finalmente no fueron necesarios los 800 pacientes ya que la tasa de no respuesta fue muy por debajo de lo esperado (20\%). El estudio de la "no respuesta" no arrojó sesgo de selección). La tasa de participación fue de un $95 \%$.

La mayoría de los encuestados fueron mujeres (de cada 5 personas que consultaron 3 eran de sexo femenino), solo el $40 \%$ estaba casado/a o 
en unión libre, 32,6 \% tenían entre 18 a 34 ańos de edad, el 53,3\% de los individuos tenían entre 35 a 64 años y 14,1\% tenían más de 65 años de edad. El promedio de edad de la población relevada fue de 44 años $( \pm 16,8)$ (Tabla 1).

Las características socio económicas de la población estudiada indicaron que: tres cuartas partes de la población tenía un nivel educativo correspondiente a primaria o secundaria y sólo un $15 \%$ tenía estudios terciarios (Tabla 1).

Considerando el acceso a los servicios que brindan atención médica, dos de cada tres participantes se atendían en la Administración de los Servicios de Salud del Estado (ASSE). Dos de cada tres sujetos manifestó que cuando tiene problemas de salud bucal recurre al sub-sector privado (mutualista, cooperativas, consultorio de salud).

\section{Factores comportamentales y metabólicos}

En relación a los factores comportamentales (Tabla 2), se observó que casi $91 \%$ de los sujetos no alcanzan a consumir la recomendación de 5 porciones de frutas y/o verduras diarias, siendo el consumo similar en los tramos etarios de 18-24 años y los 25-64 años. El 44,7\% de los sujetos presentó actividad física insuficiente. En términos relativos un 58,0\% más de individuos de mediana edad en relación a los jóvenes presentaron actividad física insuficiente.

En cuanto al hábito de fumar la diferencia mayor se encuentra entre los adultos mayores y los adultos de mediana edad, estos últimos fuman diariamente tres veces más que los primeros.

Con respecto al consumo de sal agregada los adultos presentaron un consumo de sal agregada $1 / 3$ menor que en los jóvenes.

Con respecto al consumo problemático de alcohol no se observaron diferencias importantes entre los tramos de edad (Tabla 2).

Los individuos adultos presentaron aproximadamente dos veces y media más (164\%) prevalencia de sobrepeso y obesidad respecto a los individuos más jóvenes. Mientras que el perí-
Tabla 1. Perfil sociodemográfico

\begin{tabular}{|l|l|l|l|}
\hline Sexo & N & proporción & \multicolumn{1}{|l}{ IC 95\% } \\
\hline Masculino & 250 & $41,5 \%$ & $(37,6-45,5)$ \\
\hline Femenino & 352 & $58,5 \%$ & $(54,5-62,4)$ \\
\hline Edad & & & \\
\hline menos de 34 & 196 & $32,6 \%$ & $(28,8-36,3)$ \\
\hline de 35 a 44 & 113 & $18,8 \%$ & $(15,7-21,9)$ \\
\hline de 45 a 54 & 100 & $16,6 \%$ & $(13,6-19,6)$ \\
\hline de 55 a 64 & 108 & $17,9 \%$ & $(14,9-21)$ \\
\hline 65 o mas & 85 & $14,1 \%$ & $(11,3-16,9)$ \\
\hline Escolaridad & & & \\
\hline hasta primaria & 172 & $28,6 \%$ & $(25-32,2)$ \\
\hline hasta ciclo básico & 175 & $29,1 \%$ & $(25,4-32,7)$ \\
\hline hasta bachillerato & 162 & $26,9 \%$ & $(23,4-30,5)$ \\
\hline hasta terciaria & 93 & $15,4 \%$ & $(12,6-18,3)$ \\
\hline Ingresos (miles) & & & \\
\hline hasta 17 & 325 & $55,4 \%$ & $(51,4-59,3)$ \\
\hline de 17 a 24 & 109 & $18,6 \%$ & $(15,5-21,7)$ \\
\hline de 24 a 32 & 80 & $13,6 \%$ & $(10,9-16,4)$ \\
\hline más de 32 & 58 & $9,9 \%$ & $(7,5-12,3)$ \\
\hline no sabe/se rehúsa & 15 & $2,6 \%$ & $(1,3-3,8)$ \\
\hline Cobertura odontológica gratuita & \\
\hline No & 404 & $67,3 \%$ & $(63,6-71,1)$ \\
\hline Si & 196 & $32,7 \%$ & $(28,9-36,4)$ \\
\hline Cobertura de salud & & & \\
\hline Mutualista & 169 & $28,1 \%$ & $(24,5-31,7)$ \\
\hline ASSE & 406 & $67,4 \%$ & $(63,7-71,2)$ \\
\hline Otros & 27 & $4,5 \%$ & $(2,8-6,1)$ \\
\hline
\end{tabular}

metro de cintura de riesgo (PCR) fue de 39,4\% a nivel global, la prevalencia relativa en individuos adultos con respecto a los más jóvenes fue de 4,4 (340\%).

Los individuos adultos con hipertensión arterial fueron casi 2 veces más que los hipertensos más jóvenes.

En cuanto a la diabetes se observa que la prevalencia de 15,6\%, aumentando con la edad. Los adultos tuvieron 3 veces más prevalencia que los jóvenes (Tabla 2). 


\section{Discusión}

El presente estudio toma una población específica de pacientes que demandan atención odontológica en la Facultad de Odontología en el período 2015-2016, siendo el primer estudio nacional que intenta determinar conjuntamente la prevalencia de los factores de riesgo de las ENT y las enfermedades bucales más frecuentes. Se obtuvo un alto grado de participación (95\%). Se constató una elevada prevalencia de inactividad física, ingesta problemática de alcohol, consumo excesivo de sal, hipertensión y diabetes. Estas cifras fueron mayores a las encontradas a nivel nacional.
El perfil sociodemográfico de los sujetos que llegan a la Facultad buscando atención, se caracteriza por: ser adultos en su amplia mayoría de 18 a 64 años, poseer como nivel de instrucción más elevado la educación primaria, contar con un nivel de ingresos que no superan los 3 salarios mínimos y cuya cobertura en salud general se realiza en el sub-sector público (70\%). Esta proporción es casi inversa a la que correspondía a las características de la población uruguaya en su conjunto donde la proporción de individuos que resolvía su atención a la salud general, en el ańo en el cual se realizó el presente relevamiento, en el sector público era aproximadamente un $30 \%{ }^{(17)}$ Para el caso de la atención a la salud bucal, los sujetos declaran acudir al sector pri-

Tabla 2. Perfil de factores metabólicos y comportamentales

\begin{tabular}{|c|c|c|c|c|c|}
\hline & $\begin{array}{l}\text { Prevalencia Total } \\
\text { (IC 95) }\end{array}$ & $\begin{array}{l}\text { Prevalencia de } 18 \\
\text { a } 24 \text { años (IC } 95)\end{array}$ & $\begin{array}{l}\text { Prevalencia de } 25 \\
\text { a } 64 \text { años (IC } 95)\end{array}$ & $\begin{array}{l}\text { Prevalencia de más } \\
\text { de } 65 \text { años (IC 95) }\end{array}$ & $\begin{array}{c}\text { Relación entre } \\
\text { adultos de } 25-64 \text { y } \\
\text { jóvenes de 18-24 }\end{array}$ \\
\hline $\mathrm{N}$ & 602 & 88 & 428 & 86 & --- \\
\hline \multicolumn{6}{|c|}{ Factores Comportamentales } \\
\hline $\begin{array}{l}\text { Consumo de frutas y verdu- } \\
\text { ras Insuficiente }\end{array}$ & $\begin{array}{c}90,8 \% \\
(88,3-93)\end{array}$ & $\begin{array}{c}89,7 \% \\
(81,3-95,2)\end{array}$ & $\begin{array}{c}91,8 \% \\
(88,8-94,2)\end{array}$ & $\begin{array}{c}87,2 \% \\
(78,3-93,4)\end{array}$ & 1,02 \\
\hline $\begin{array}{l}\text { Consumo de sal agregada } \\
\text { a las comidas Siempre/Casi } \\
\text { siempre }\end{array}$ & $\begin{array}{c}17,7 \% \\
(14,7-21)\end{array}$ & $\begin{array}{c}26,1 \% \\
(17,3-36,6)\end{array}$ & $\begin{array}{c}17,2 \% \\
(13,7-21,1)\end{array}$ & $\begin{array}{c}11,6 \% \\
(6,4-20,1)\end{array}$ & 0,66 \\
\hline Consumo diario de tabaco & $\begin{array}{c}26,7 \% \\
(23,2-30,5)\end{array}$ & $\begin{array}{c}20,5 \% \\
(12-31,6)\end{array}$ & $\begin{array}{c}32,2 \% \\
(27,8-36,9)\end{array}$ & $\begin{array}{c}9,3 \% \\
(4,8-17,3)\end{array}$ & 1,57 \\
\hline Actividad física insuficiente & $\begin{array}{c}44,7 \% \\
(40,7-48,8)\end{array}$ & $\begin{array}{c}29,5 \% \\
(20,3-40,2)\end{array}$ & $\begin{array}{c}46,7 \% \\
(41,9-51,6)\end{array}$ & $\begin{array}{c}50,0 \% \\
(39,0-61,0)\end{array}$ & 1,58 \\
\hline $\begin{array}{l}\text { Consumo problemático de } \\
\text { alcohol }\end{array}$ & $\begin{array}{c}15,8 \% \\
(18,2-24,7)\end{array}$ & $\begin{array}{c}19,8 \% \\
(12-29,8)\end{array}$ & $\begin{array}{c}14,8 \% \\
(11,6-18,6)\end{array}$ & $\begin{array}{c}16,3 \\
(9,2-25,8)\end{array}$ & 0,75 \\
\hline \multicolumn{6}{|c|}{ Factores Metabólicos } \\
\hline Sobrepeso-Obesidad & $\begin{array}{c}57,1 \% \\
(53-61,1)\end{array}$ & $\begin{array}{c}22,7 \% \\
(14,5-32,9)\end{array}$ & $\begin{array}{c}60 \% \\
(56,6-64,4)\end{array}$ & $\begin{array}{c}76,7 \% \\
(66,4-85,2)\end{array}$ & 2,64 \\
\hline $\begin{array}{l}\text { Perímetro de Cintura de } \\
\text { Riesgo }\end{array}$ & $\begin{array}{c}39,4 \% \\
(35,4-43,3)\end{array}$ & $\begin{array}{c}13,6 \% \\
(10,9-16,4)\end{array}$ & $\begin{array}{c}40,4 \% \\
(36,5-44,3)\end{array}$ & $\begin{array}{c}60,5 \% \\
(56,5-64,4)\end{array}$ & 2,97 \\
\hline Hipertensión & $\begin{array}{c}40 \% \\
(36,1-44,1)\end{array}$ & $\begin{array}{c}19,3 \% \\
(11,7-29,1)\end{array}$ & $\begin{array}{c}37,6 \% \\
(33-42,4)\end{array}$ & $\begin{array}{c}73,3 \% \\
(62,6-82,2)\end{array}$ & 1,95 \\
\hline Diabetes & $\begin{array}{c}15,6 \% \\
(12,5-18,8)\end{array}$ & $\begin{array}{c}4,5 \% \\
(1,8-11,1)\end{array}$ & $\begin{array}{c}15,0 \% \\
(11,9-18,6)\end{array}$ & $\begin{array}{c}30,2 \% \\
(21,5-40,6)\end{array}$ & 3,33 \\
\hline
\end{tabular}


vado(66\%), en el caso de poder afrontar los gastos que ello supone. Esto pone en evidencia que la Facultad recibe sujetos que constituyen un sector muy vulnerado de la sociedad uruguaya que no están siendo amparados en su derecho a la salud bucal como sería de esperar de acuerdo a la ley 18211 del Sistema Nacional Integrado de Salud, ley que se ve limitada de acuerdo al catálogo de prestaciones (PIAS) previsto para la salud bucal ${ }^{(18,19)}$.

Con respecto a los factores comportamentales el consumo insuficiente de frutas y verduras y el hábito de fumar tuvieron un valor similar a los resultados de la $2 \mathrm{da}$ encuesta nacional de los factores de riesgo de las ENT (2a ENFRENT) (11) tanto en la muestra global como en los ambos tramos etarios.

Por otro lado, el consumo de sal agregada a las comidas para el total de la población del presente estudio fue $17,7 \%$ mientras que en la $2 \mathrm{a} E N-$ FRENT fue $14,1 \%$,presentando un incremento relativo del $26 \%$ de individuos consumidores de sal agregada en la población de la Facultad de Odontología. Este consumo en el tramo más joven en el presente estudio fue de $26,1 \%$ mientras que a nivel nacional fue de 19,3\% evidenciando un aumento en la prevalencia relativa de los consumidores frecuentes de sal de un 35\% a favor de la población del presente estudio. En el tramo de 25 a 64 años el sobreagregado de sal a las comidas fue de $17,2 \%$, mientras que a nivel nacional fue de 12,5\% (37\% más en los pacientes de FO). La frecuencia más elevada en ambos tramos de edad en el presente estudio podría explicar en cierta medida la mayor prevalencia global de hipertensos respecto a la 2a ENFRENT. En igual sentido al analizar la actividad física insuficiente globalmente encontramos una mayor proporción de individuos en el presente estudio: $44,7 \%$ entretanto a nivel nacional fue de solo un 21,6\%, mostrando 2,1 veces más prevalencia relativa de individuos con actividad física insuficiente en favor del presente estudio.
Finalmente al analizar el consumo problemático del alcohol y la actividad física insuficiente,sin discriminar el tramo de edad, encontramos un incremento del doble en este estudio comparado con la población nacional.

$\mathrm{Al}$ considerar los factores de riesgo metabólicos, encontramos que la prevalencia de sobrepeso y obesidad (IMC $>25 \mathrm{~kg} / \mathrm{m} 2$ ) en el total de la población de nuestro estudio es muy similar a la hallada a nivel nacional: $57,1 \%$ y $58,5 \%$ respectivamente, siendo también similar la prevalencia de obesidad en el tramo etario de 25 a 64 ańos. Al mismo tiempo, también es similar la prevalencia de perímetro de cintura de riesgo en la población del presente estudio respecto a los datos de la 2a ENRENT respectivamente. Considerando el sobrepeso - obesidad para el tramo etario más joven, la proporción de jóvenes con este factor de riesgo en la población de este estudio es menor que la reportada a nivel nacional (40\% menor). Queda la interrogante si se trata de una población joven más sana con respecto a sobrepeso y obesidad, lo cual debería ser confirmado.

La hipertensión presentó una prevalencia relativa mayor en pacientes de FO del 34\% con respecto a nivel nacional, siendo de $40 \%$ y de $29,8 \%$ respectivamente. Mientras que en el tramo de edad de 25 a 64 años presenta valores similares a los valores nacionales, en el tramo más joven la prevalencia relativa de hipertensos es 2, 2 veces más en los pacientes de FO que los valores de la 2a ENRENT.

La presencia de diabetes en este estudio fue definida por la glicemia capilar mayor a 200mg/ $\mathrm{dl}$ (con o sin ayuno) o por el autoreporte de diabetes cuando esta fue diagnosticada por un médico. En la $2^{\text {a }}$ ENRENT la diabetes se consideró cuando la glicemia de ayuno era igual o superior a $126 \mathrm{mg} / \mathrm{dl}$ o por autoreporte de ser diabético o estar en tratamiento medicamentoso por diabetes. La prevalencia para el grupo de edad entre 25 y 64 años fue de 15,6 (40\% mayor) en la presente encuesta con respecto a la encuesta nacional. Las diferencias en los crite- 
rios utilizados podrían explicar la mayor prevalencia de diabetes en la población relevada en el presente estudio.

Debe considerarse que al tratarse de una población específica que demanda atención odontológica en una institución de enseñanza universitaria sin fines de lucro, tanto las características socio demográficas como el perfil de las condiciones bucales encontradas son una limitante para comparar la misma con estudios de base poblacional.

\section{Conclusiones}

La elevada prevalencia de factores de riesgo comportamentales: insuficiente actividad física, ingesta problemática de alcohol y consumo excesivo de sal agregada en las comidas, así como de factores de riesgo metabólicos: hipertensión y diabetes en la población estudiada en relación a las prevalencias existentes a nivel nacional, permitió detectar la necesidad de un abordaje integrado de la salud diseñando programas comunes para personas que concurren a la Facultad de Odontología, que apunten a la promoción de hábitos saludables así como preventivos de las enfermedades no transmisibles y de las enfermedades bucales y que puedan ser utilizados en otros centros asistenciales.

\section{Contribución de autoría}

1. Concepción y diseño del estudio

2. Adquisición de datos

3. Análisis de datos

4. Discusión de los resultados

5. Redacción del manuscrito

6. Aprobación de la versión final del manuscrito
S.M.L.E ha contribuido en 1, 3, 4, 5, 6 .

E.S ha contribuido en 1, 3, 4, 5, 6 .

M.M ha contribuido en 2, 6 .

P.M ha contribuido en 2, 6 .

R.A ha contribuido en 1, 3, 4, 6 .

A.F ha contribuido en 2, 3, 4, 5, 6 .

F.M ha contribuido en 3, 4, 6 .

P. M ha contribuido en 3, 4, 6 .

B.V ha contribuido en 2, 6 .

S.P ha contribuido en 2, 6 .

L. C ha contribuido en 2, 6 .

F.P ha contribuido en 2 .

R.P ha contribuido en 2 .

R.S ha contribuido en 2 .

M.G ha contribuido en 2 .

\section{Referencias}

1. Misa A. Demografía. En: Benia W, Reyes I. (Coord). Temas de salud pública Montevideo. Montevideo: Oficina del libro FEFMUR; 2009. p. 17-46.

2. Dever A, Champagne F. Epidemiologia y Administración de Servicios de Salud. Organización Panamericana de la Salud. 1991. 417 p.

3. Jaime Breilh. Debates y desafíos. Epidemiología crítica latinoamericana: raíces, desarrollos recientes y ruptura metodológica. En: Carolina Morales, Juan Carlos Eslava. Tras las huellas de la determinación. 1era. ed. Bogotá: Universidad Nacional de Colombia; 2014. p. 17-77

4. WHO. Non communicable diseases. Fact sheet updated Junio 2018. [en linea] [ consultado 30 de octubre de 2018] Disponible en: http: // www.who.int/mediacentre/factsheets/fs355/en/

5. Uruguay. Ministerio de Salud Pública. Enfermedades No Transmisibles. [en línea] [ consultado 30 de octubre de 2018] Disponible en: http: //www.msp.gub.uy/noticia/enfermedades-notransmisibles

6. Hosseinpoor AR, Bergen N, Kunst A, Harper S, Guthold R, Rekve D, d'Espaignet ET, Naidoo N, Chatterji S. Socioeconomic inequalities in risk factors for non communicable diseases in low-income and middle-income countries: 
results from the World Health Survey. BMC Public Health. 2012; 12: 912

7. World Health Organization. Global Action Plan for the prevention and control of noncommunicable diseases 2013-2020. [en línea] Disponible en http: //apps.who.int/iris/bitstream/ handle/10665/94384/9789241506236_eng. pdf?sequence $=1$

8. Petersen PE, Ogawa H. The Global Burden of periodontal disease: Towards integration with chronic disease prevention and control. 2012. Periodontol 2000. 2012; 60 (1): 15-39.

9. Oral Disease Added to United Nations Declaration on Non communicable Diseases Essential Dental Knowledge. J Can Dent Assoc 2011; 77: b138. Disponible en: http: //www.jcda.ca/ article/b138.

10. Watt RG. Social determinants of oral health inequalities: implications for action. Community Dent Oral Epidemiol. 2012; 40 (supp.2): 44-48.

11. Rubinstein AL, Irazola VE, Calandrelli M, Rubinstein A, Irazola V,* Calandrelli M, Elorriaga N, Gutierrez L, Lanas F, Manfredi J, Mores N, Olivera H, Poggio R, Ponzo J, Seron P, Chen C, Bazzano LA,Hef J. Multiple cardiometabolic risk factors in the Southern Cone of Latin America: a population-based study in Argentina, Chile, and Uruguay. Int J Cardiol. 2015; 183: 82-88. doi: 10.1016/j.ijcard.2015.01.062

12. MSP, Segunda Encuesta Nacional de Factores de Riesgo de Enfermedades No Transmisibles. PPENT. Montevideo, 2013. [en línea] [consultado 30 de octubre de 2018] Disponible en: http: //www.msp.gub.uy/ publicaci $\% \mathrm{C} 3 \% \mathrm{~B} 3 \mathrm{n} / 2 \% \mathrm{C} 2 \% \mathrm{AA}$-encuesta-na- cional-de-factores-de-riesgo-de-enfermedadesno-transmisibles

13. World Health Organization. Oral Health Surveys. Basic Methods. 4th Edition [Internet]. Geneva; 2013.

14. Lorenzo S. Editorial: Primer Relevamiento Nacional de Salud Bucal en población joven y adulta uruguaya [Internet]. [citado 2014 May 21]. Disponible en: http: //www.scielo.edu.uy/ pdf/ode/v15nspe/v15nspea02.pdf

15. Lorenzo S, Álvarez R, Blanco S, Peres M. Primer Relevamiento Nacional de Salud Bucal en población joven y adulta uruguaya: Aspectos metodológicos. Odontoestomat. 2013; 15( spe ): 8-25.

16. WHO. Obesity: preventing and managing the global epidemic. Report of a WHO Consultation. WHO Technical Report Series 894

17. Oreggioni I. El camino hacia la cobertura universal en Uruguay: cobertura poblacional del Sistema Nacional Integrado de Salud. En: Economìa, política y economía política para el acceso y la cobertura universal de salud en el Uruguay. OPS 2015. Disponible en: https: // www.paho.org/uru/index.php?option=com_ docman \&view = download \& category slug=publicaciones-sistemas-y-servicios-desalud\&alias $=468$ - capitulo-2-pdf\&Itemid $=307$

18. Uruguay 2007. Poder Legislativo. Ley No 18.211 SISTEMA NACIONAL INTEGRADO DE SALUD. Disponible en: https: // legislativo.parlamento.gub.uy/temporales/leytemp28458.htm

19. Uruguay 2008. Ministerio de Salud Pública. Plan Integral de Atención en Salud (PIAS). Disponible en: http: //www.msp.gub.uy/sites/ default/files/Decreto_y_Anexo_I.pdf

Susana Margarita Lorenzo: serepistat@gmail.com 